\title{
Does the offer of cervical screening with breast screening encourage older women to have a cervical smear test?
}

\author{
Gillian Lancaster, Peter Elton
}

\begin{abstract}
Objective-The aim was to determine what effect the offer of a cervical smear test when attending for breast screening has on the uptake of cervical and breast screening.

Design-The study involved randomisation to compare uptake in those women invited for cervical screening in advance with their breast screening invitation (group 1) with those invited for breast screening only and then offered a smear test upon arrival for breast screening (group 2). The main outcome measure was improvement in the uptake of cervical screening among older women without detriment to the breast screening service.
\end{abstract}

Setting-The study took place at the Northern Hospital in North Manchester.

Participants-Participants were 2131 women aged 50-64 years invited for breast screening at the Northern Hospital in the summer of 1990 .

Main results-Overall, $54 \%$ of the women who were eligible attended for breast screening, $52 \%$ attended from group 1 and $55 \%$ from group 2 . Of those attending for breast screening, 957 were eligible for cervical screening and $193(20 \%)$ had a smear test. There was a difference in the proportion tested from each group ( $p<0.001), 28 \%$ had a smear test from group 1 and $13 \%$ from group 2. Forty five percent of the $193 \mathrm{had}$ not had a cervical smear for at least five years.

Conclusions-The cervical screening facility did attract some women who were overdue for a smear test and who might not normally have attended for cervical screening, and there was no evidence to suggest that it had a detrimental effect on the breast screening uptake. An advanced cervical screening invitation seemed preferable to an invitation upon arrival at the breast screening unit.

f Epidemiol Community Health 1992; 46: 523-527 Christie Hospital NHS Trust, Kinnaird Road, Withington,

Manchester M20 9QL,

United Kingdom

G Lancaster

North Manchester

District Health

Authority, Central

Drive, Crumpsall,

Manchester, United

Kingdom

P Elton

Correspondence to:

Mrs Lancaster

Accepted for publication March 1992

Destrodion of a computerised call scheme for cervical screening population coverage, particularly among high risk women, has been disappointing. ${ }^{1}$ Recent studies have shown that older women are the least likely to have had a cervical smear, especially those from lower socioeconomic groups. ${ }^{2-4}$

The introduction of computer managed breast screening services, specifically aimed at older women in the 50-64 year age band, may provide uptake in this group of women. Certainly the response to breast screening so far suggests that older women are willing to use this facility ${ }^{5}$ and it is possible that cervical screening would be more acceptable when offered in the context of other services. Moreover, recent guidelines on breast cancer screening from the Department of Health Advisory Committee ${ }^{6}$ state that wherever possible there should be cooperation between cervical and breast screening services.

During a study ${ }^{7}$ carried out by the Edinburgh breast screening unit, 446 women attending for breast screening completed a questionnaire about their smear history. Overall, $29 \%$ of the women had never had a smear and $63 \%$ had not been tested in the last five years. In a further study ${ }^{7}$ at the unit, women were offered a cervical smear at two clinics held weekly, while attending for breast screening. Among the 323 women attending the clinic, $127(39 \%)$ had a smear test, $14(4 \%)$ women wanted a test but could not have one for various reasons, $135(42 \%)$ did not want to have a smear test, and 47 women $(15 \%)$ were found to be ineligible for cervical screening. Of the women wanting a smear, $43 \%$ found it convenient that this service was available, and of those not wanting a smear, $47 \%$ had had one recently.

In North Manchester a preliminary study showed that out of 2595 women attending for breast screening, 398 women $(15 \%)$ took the opportunity to have a cervical smear at the same time, some being invited by post for cervical screening with the breast screening invitation, others upon arrival at the unit. Of these 398 women, 277 had not had a smear in the past five years and 70 did not recall ever having had a test. However, there was some question as to whether the invitation for cervical screening had had a detrimental effect on the uptake of breast screening.

The aims of this study were twofold: (1) to determine what effect the inclusion of an advanced invitation for a cervical smear along with the breast screening invitation had on the uptake of cervical screening, when compared with the offer of a cervical smear upon arrival at the breast screening unit; and (2) to compare the effect of the two approaches on response to the breast screening service. the opportunity to encourage cervical screening

\section{Methods}

All eligible women registered with 10 general practitioner practices in North Manchester and other fringe practices lying on the outskirts of North Manchester, were selected by Manchester Family Health Services Authority (FHSA) to be invited for breast screening, when the mobile 
breast screening unit was located at the Northern Hospital in North Manchester between 25th June and 8th August 1990. Each general practitioner was sent a list giving prior notification of women selected from their practice for breast screening to check their eligibility. Once the information from the general practitioners had been processed, the FHSA notified the breast screening office, situated in the Nightingale Centre at Withington Hospital, South Manchester, of the women to be invited.

At the Nightingale Centre the women were separated into nine batches ready for invitation, grouping together general practitioners or practices. The women were randomly allocated into two groups. Group 1 consisted of those women who were sent a cervical screening invitation with the breast screening invitation, which gave an appointment time for attending the mobile unit. Group 2 contained those women who were sent a breast screening invitation only. Both groups, upon arrival at the mobile unit, were given a leaflet offering the opportunity to have a cervical smear.

Breast screening appointments were allocated automatically by computer and the invitation letters sent out in batches approximately three weeks before the appointment dates. Translated letters were sent to all women with Asian names in addition to the English letters. The cervical screening invitation letters for those women in group 1 were included with the first round of breast screening invitations only and not with any subsequent letters to non-attenders.

Facilities to have a smear test were set up in the outpatients department of the Northern Hospital, close to the mobile unit, for the course of the study. Before or after breast screening, women could go without an appointment to have a smear taken by a female nurse. It was suggested in the invitation letter and leaflet that the cervical smear test could be taken by their general practitioner instead, if so desired.

Throughout the study all enquiries and telephone calls about the cervical smear test were monitored to record the nature of the enquiries, and to determine the additional workload on the staff involved in offering the cervical screening facility. Calls or enquiries could be made to the Nightingale Centre, the mobile unit, the nurse at the cervical screening clinic in the Northern Hospital, or a special telephone number given in the cervical screening invitation letter and leaflet. This telephone line was managed by the treatment room nurses at North Manchester's Ancoats community clinic, who were mainly responsible for taking the cervical smears at the Northern Hospital throughout the study period.

At the end of the screening period, the batch lists were printed out at the Nightingale Centre with the results of the breast screening. The cervical screening response and results were

Table I Response to breast screening by randomisation group

\begin{tabular}{lllc}
\hline & $\begin{array}{l}\text { Sent cervical screening } \\
\text { invitation in advance }(\%)\end{array}$ & $\begin{array}{l}\text { Not sent } \\
\text { invitation }(\%)\end{array}$ & Total (\%) \\
\hline $\begin{array}{llll}\text { Attended for } \\
\text { breast screening }\end{array}$ & $506(52)$ & $519(55)$ & $1025(54)$ \\
Did not attend & $459(48)$ & $428(45)$ & $887(46)$ \\
Total & 965 & 947 & 1912 \\
\hline
\end{tabular}

recorded by the nurses who took the smears. Any smear tests taken by general practitioners were also monitored.

\section{Results}

BREAST SCREENING

Of the 2131 women invited for breast screening, $1025(48 \%)$ attended and were screened and 887 $(42 \%)$ did not attend. The remaining $219(10 \%)$ women were found to be ineligible for screening; 183 women had moved out of the area, nine had died, five had been screened recently, and 22 had varied reasons for not attending, the majority being ill.

Table I shows the response of the 1912 eligible women to the breast screening invitations compared with whether or not they were sent an advanced invitation for cervical screening. Although 3\% more women in the second group attended for breast screening, this was not statistically significant.

The mean age of the women in both group 1 and group 2 was 56 years, whether including or excluding ineligible women. The mean ages of those responding to breast screening and those who did not attend were compared within each randomisation group by using analysis of variance. The results of the analysis showed no evidence to suggest any difference in the ages of the responders and non-responders, whether they were sent an advanced cervical screening invitation or not.

In total, the women in the study were registered with 57 general practitioners. The practitioners were separated into 11 groups made up of 10 main practices (practices A to J), plus a group containing another 28 practitioners (practice $\mathrm{K}$ ) from fringe practices on the outskirts of the screening area. As table II indicates, there was a significant difference in the uptake of breast screening by the women registered within these practice groups

Table II Response to breast screening by general practitioner (GP) practice

\begin{tabular}{lcrr}
\hline GP practice $(\%)$ & $\begin{array}{l}\text { Attended for } \\
\text { breast screening }\end{array}$ & Did not attend & Total \\
\hline A & $208(53)$ & $187(47)$ & 395 \\
B & $141(72)$ & $55(28)$ & 196 \\
C & $19(36)$ & $34(64)$ & 53 \\
D & $67(64)$ & $37(36)$ & 104 \\
E & $145(53)$ & $127(47)$ & 272 \\
F & $163(58)$ & $117(42)$ & 280 \\
G & $43(42)$ & $60(58)$ & 103 \\
H & $84(44)$ & $107(56)$ & 191 \\
I & $87(62)$ & $54(38)$ & 141 \\
J & $37(33)$ & $75(67)$ & 112 \\
K & $31(48)$ & $34(52)$ & 65 \\
Total & $1025(54)$ & $887(46)$ & 1912 \\
\hline \multicolumn{4}{c}{$\left(\chi_{10}^{2}=77 \cdot 27, \mathrm{p}<0.001\right)$} \\
\end{tabular}

$(p<0.001)$. Practices C, G, H, and J had a substantially higher proportion of non-responders to breast screening. In practices $\mathrm{G}$ and $\mathrm{J}$, approximately $60 \%$ of the women in the study were Asian, whereas in the other nine practice groups the Asian women only accounted for between $1 \%$ and $6 \%$ of the women.

The high proportion of Asian women with poorer attendance was examined in more detail to determine whether this influenced the breast screening figures. The 1912 women were separated into Asian and non-Asian by surname by an 
Table III Response to breast screening by Asian and non-Asian women within randomisation groups

\begin{tabular}{|c|c|c|c|c|c|c|}
\hline & \multicolumn{3}{|l|}{ Asian } & \multicolumn{3}{|l|}{ Non-Asian } \\
\hline & 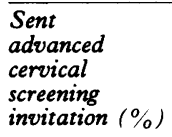 & $\begin{array}{l}\text { Not sent } \\
\text { invitation }(\%)\end{array}$ & Total $(\%)$ & $\begin{array}{l}\text { Sent } \\
\text { advanced } \\
\text { cervical } \\
\text { screening } \\
\text { invitation }(\%)\end{array}$ & $\begin{array}{l}\text { Not sent } \\
\text { invitation }(\%)\end{array}$ & Total $(\%)$ \\
\hline $\begin{array}{l}\text { Attended for } \\
\text { breast screening } \\
\text { Did not attend }\end{array}$ & $\begin{array}{l}25(29) \\
61(71)\end{array}$ & $\begin{array}{l}32(37) \\
54(63)\end{array}$ & $\begin{array}{r}57(33) \\
115(67)\end{array}$ & $\begin{array}{l}481(55) \\
398(45)\end{array}$ & $\begin{array}{l}487(57) \\
374(43)\end{array}$ & $\begin{array}{l}968(56) \\
772(44)\end{array}$ \\
\hline Total & 86 & 86 & 172 & 879 & 861 & 1740 \\
\hline
\end{tabular}

Urdu interpreter. Overall on this basis $172(9 \%)$ of the eligible women were Asian. There was a significant difference in the response to breast screening $\left(\chi_{1}^{2}=30.94, \mathrm{p}<0.001\right)$ between the two groups. As shown in table III, $33 \%$ of the Asian women attended for breast screening, whereas $56 \%$ of the non-Asian women were screened. When the Asian women were excluded from the analysis, the percentage of women responding to the breast screening invitation was $55 \%$ for group 1 women, who were sent an advanced cervical screening letter, compared to $57 \%$ for group 2 women, who were not sent a letter in advance. The proportion of Asian women responding to the invitation was $29 \%$ in group 1 and $37 \%$ in group 2, a larger difference indicating that the Asian women might have been put off attending for breast screening if invited for cervical screening at the same time, although the difference was still not statistically significant.

Table IV Cervical screening history of those women attending for breast screening who were eligible for cervical screening

\begin{tabular}{lccc}
\hline & $\begin{array}{c}\text { Had a smear } \\
\text { test }(\%)\end{array}$ & $\begin{array}{l}\text { Did not have } \\
\text { smear test }(\%)\end{array}$ & Total $(\%)$ \\
\hline Previous smear in last 5 years & $107(55)$ & $583(76)$ & $690(72)$ \\
Previous smear 5-10 years ago & $29(15)$ & $40(5)$ & $69(7)$ \\
Previous smear over 10 years ago & $14(7)$ & $15(2)$ & $29(3)$ \\
No cervical smear record & $43(22)$ & $126(16)$ & $169(18)$ \\
Total & 193 & 764 & 957 \\
\hline
\end{tabular}

CERVICAL SCREENING

The cervical screening histories of the women were found, initially, from information held on the FHSA computer, which has recorded all smears for North Manchester residents since January 1986 when the call/recall system was introduced. If no details were found then the woman's history was traced from the North Western Regional Cytology Laboratory computer.

In 118 cases the general practitioner had cancelled further smear tests, mainly because the women had had a hysterectomy, making these women ineligible for cervical screening. Of the 1794 women in the study eligible for cervical screening, $967(54 \%)$ had had a smear test in the past five years, $111(6 \%)$ had been tested between five and 10 years ago, and $82(5 \%)$ women had not had a smear test for more than 10 years. No previous smear information was found for 634

Table $V$ Response to cervical screening by randomisation group for those women who attended for breast screening and were eligible for cervical screening

\begin{tabular}{llcl}
\hline & $\begin{array}{l}\text { Sent cervical screening } \\
\text { invitation in advance }(\%)\end{array}$ & $\begin{array}{l}\text { Not sent } \\
\text { invitation }(\%)\end{array}$ & Total $(\%)$ \\
\hline $\begin{array}{l}\text { Had a smear test } \\
\text { Did not have a }\end{array}$ & $131(28)$ & $62(13)$ & $193(20)$ \\
$\begin{array}{l}\text { smear test } \\
\text { Total }\end{array}$ & $343(72)$ & $421(87)$ & $764(80)$ \\
\hline & 474 & 483 & 957 \\
\hline
\end{tabular}

women $(35 \%)$. Twenty six $(1 \%)$ of the women had been removed from the FHSA register since the end of the study, most because they had moved out of the area and so no information was available on the FHSA computer. These women were also not found on the cytology laboratory computer. The remaining $608(34 \%)$ women had no smear tests recorded on either computer system.

The fact that no computer records were found for some women does not necessarily mean that the women had never been screened. The FHSA computer had only held cytology information for four and a half years at the time of the study, and the cytology laboratory computer did not process every smear taken in the region or those taken outside the region. Neverthless, it is probable that the women had not been tested for at least four and a half years.

During the breast screening period, $195(11 \%)$ of the 1794 eligible women in the study attended the cervical screening clinic. Two of the women did not keep their breast screening appointment but still had a smear test at the clinic. Only seven of the 195 women were Asian.

Table IV summarises the cervical screening histories of the 957 women who attended for breast screening and were eligible for cervical screening ( 68 women were found to be ineligible because they had had a hysterectomy), broken down by those who had a smear test and those who did not. Of the 267 women attending for breast screening who had not had a smear test in the past five years, $86(32 \%)$ had a test at the clinic-62 $(42 \%)$ of the 146 women in group 1 and $24(20 \%)$ of the 121 women in group 2. Among the 837 women eligible for cervical screening who did not attend for breast screening, only $33 \%$ had had a smear test in the past five years.

The mean age of the women attending the clinic was 56 years. Table $\mathrm{V}$ shows the number of women in each randomisation group who had a cervical smear test out of the 957 women who attended for breast screening and were eligible for cervical screening. In group 1, $131(28 \%)$ of the women who were sent a cervical screening invitation letter in advance had a cervical smear, whereas only $62(13 \%)$ of those in group 2 who were not invited in advance attended the clinic, which is a significant difference $(p<0.001)$.

Table VI lists the results of the smear tests. The majority of the women $(89 \%)$ had a normal smear. Only one woman showed signs of borderline changes and three women had an infection. Of the remaining 17 slides, nine $(5 \%)$ were too inadequate for the laboratory to read and eight $(4 \%)$ of the slides had been broken or lost in the post!

In the cervical screening invitation letter and leaflet it was mentioned that women could have a smear test with their general practitioner instead 
Table VI Results of the cervical smear tests

\begin{tabular}{lrl}
\hline & \multicolumn{3}{c}{ No of women $(\%)$} \\
\hline Normal & 174 & $(89)$ \\
Inadequate & 9 & $(5)$ \\
Infection & 3 & $(1 \cdot 5)$ \\
Wart virus/ & 1 & $(0 \cdot 5)$ \\
borderline changes & 8 & $(4)$ \\
$\begin{array}{l}\text { Slide broken or lost } \\
\text { in post }\end{array}$ & 195 & \\
Total & & \\
\hline
\end{tabular}

of at the clinic if they wished. For this reason, smear tests taken by general practitioners during the study period were monitored. All smear tests taken over a period of eight weeks from the approximate time that the woman received her cervical screening invitation letter or leaflet were counted. For women in group 1 , who were sent a cervical screening invitation letter in advance with the breast screening invitation approximately three weeks before breast screening, smear tests were counted from the dates on which the letters were sent out. However, since the dates on which the women attended for breast screening were not available, the dates on which the breast screening invitation letters were sent out were used again for those women in group 2, who were given an invitation leaflet upon arrival for breast screening, but an extra three weeks were allowed to account for the time between receiving the letter and attending for the breast screening appointment.

During the given time periods 45 women had a smear test at their general practitioner's surgery. However, 12 of these women, who were in group 2 and did not receive an advanced cervical screening invitation, did not attend for breast screening and therefore did not directly receive any literature from the study which could have prompted them to have the cervical smear. The remaining 33 women did receive an invitation for cervical screening, 20 in advance and 13 upon arrival for breast screening, but the smear tests could still have been as a result of call/recall, symptoms, or for other opportunistic reasons. Twenty five of the women attended for breast screening.

Therefore, in total $240(13 \%)$ of 1794 eligible women had a smear test during the course of the study $-151(17 \%)$ of the 908 women in group 1 and $89(10 \%)$ of the 886 women in group 2 $(p<0.001)$. This includes the two women who attended for cervical screening but not for breast screening and the 12 women who had a smear test at their general practitioner's surgery but who did not directly receive an invitation for cervical screening used in the study.

\section{MONITORING OF ENQUIRIES}

In total, the Nightingale Centre received 30 telephone calls regarding the cervical screening invitation. Eighteen calls $(60 \%)$ were immediately referred to the number at Ancoats Community Clinic. Seven women $(23 \%)$ rang to say that they did not want cervical screening, and the remaining five calls were various queries, eg, about directions and whether a female doctor would be taking the test.

Although 18 calls were referred to the number at Ancoats community clinic from the Nightingale Centre, the treatment room nurses only received six telephone calls. Three women rang to say that they did not want cervical screening and another three women had decided cervical screening was not necessary; two had recently been screened and the other was being treated at a gynaecological clinic.

Most enquiries about cervical screening were made to the radiographers on the mobile breast screening unit. The majority of the 76 enquiries $(84 \%)$ were referred immediately to the nurse in the cervical screening clinic. Eight women $\left(11_{\%}\right)$ asked for directions to the clinic and the other four women wished to discuss the smear test.

Thirty enquiries were made to the nurse at the cervical screening clinic. Fifteen $(50 \%)$ of the women had been screened recently and so were not tested again. Nine women $(30 \%)$ decided not to have a smear after discussion with the nurse for medical reasons, eg, hysterectomy. Two women were referred to a well woman clinic because they had other queries, and four women made enquiries but no details were recorded.

\section{Discussion}

The area of North Manchester in which the study took place is one of the more deprived areas of Manchester, which is reflected in the uptake of breast screening. The small non-significant difference in attendance for breast screening between those receiving an invitation for cervical screening with their breast screening letter, and those who did not, indicates that for most women there was no detrimental effect on the uptake of breast screening. It may be, though, that there was a detrimental effect on Asian women, who might have been put off attending for breast screening if invited for cervical screening at the same time. However, this was not statistically significant and the number of Asian women was only small. Further investigations are needed to support this, but there is always the problem of finding a group of Asian women large enough for such a study.

The reason for setting up a special clinic was to encourage those older women who have never been screened, or not screened within the past five years, to have a smear test. In this study, 193 $(20 \%)$ of the 957 women attending for breast screening who were eligible for cervical screening had a cervical smear test at the clinic. The majority of these women responded to an advanced invitation, and $45 \%$ had not had a smear test within the past five years. The prevalence of abnormalities in this study $(0.5 \%)$ was slightly lower than the $1.6 \%$ figure given by the regional cytology laboratory for all women aged 50-64 years (including the women in this study) whose smears were processed by the laboratory in 1990, which might reflect the extent to which these women have already been screened. The proportion of inadequate smears was also low, although there were some postal problems which have been more apparent since the introduction of new plastic boxes for transportation of the slides.

There were quite a number of enquiries about cervical screening throughout the course of the study. The special telephone line was set up to divert queries away from the breast screening centre, but this was of limited use. It might have been more appropriate to have had an extension within the breast screening centre, to which calls could immediately be transferred. Most enquiries were made to the radiographers on the mobile breast screening unit, but the majority of these 
were immediately referred to the nurse in the clinic and quite a few women were simply asking directions.

It could be argued that such opportunistic screening for a small group of women who are already in the routine call/recall system is a wasteful use of resources. However, an advanced invitation for cervical screening did attract women who were overdue for a test, and who were likely to have received previous invitations to which they had failed to respond. In the Edinburgh study ${ }^{7}$ referred to earlier, $127(46 \%)$ of the $\mathbf{2 7 6}$ women eligible for cervical screening had a smear test when attending for breast screening. Moreover, many of the women found it convenient, although there was no mention of how many had been screened recently.

The results of another study from Edinburgh ${ }^{8}$ suggested that older and less affluent women may find breast screening more acceptable if it is offered within the context of general health screening rather than emphasising the single disease. Holland ${ }^{9}$ proposed that a package of health tests should be tested for its acceptability to women. He pointed out that, for the women, it is not the site of the primary cancer that is the most predominant concern, rather it is the concept of premature death. He suggested that this might not have been officially considered, more because the convenience of those offering the service may override the preferences of the women involved.

This study has shown that it is feasible with minimal disruption to organise both breast and cervical screening services together. The combination of such services would inevitably pose administrative problems, but since most breast and cervical screening systems both use the FHSA register to identify women for invitation, it should be possible to combine the two services without too much difficulty, probably at the breast screening centres. It would take only a small amendment to the FHSA computer software to be able to flag those women who are overdue for a smear test on the invitation lists prepared for breast screening. If the breast screening software could then identify these women, they could be sent a cervical screening invitation with their breast screening letter. Thus, it would be possible to provide cervical screening as an additional service through invitations only to those older women who have not been screened within the past five years.

It would be technically feasible to integrate breast and cervical screening for all women aged 50-64 years, but there could be some concern that this would undermine the role of the general practitioner in cervical screening. It is possible that some general practitioners would welcome such a service as it would help them achieve a higher target for payment, even though it would reduce the percentage of smears that they had personally undertaken.

A combined service for those women under 50 years of age introduces other issues such as the efficacy of breast screening for this age group. Nevertheless, the idea of a general health check, including measurement of weight, blood pressure, and cholesterol level, as well as cervical screening, whether by the general practitioner or at a local health authority clinic, is still appropriate and could prove to be an attractive setting in which to place and promote the cervical smear test.

We would like to thank all the staff of the Nightingale Centre at Withington Hospital, the treatment room sisters at Ancoats community clinic, Dr R Yule and his staff at the North Western regional cytology laboratory, Manchester Family Health Services Authority, and Dr E Friedman for all their help and assistance. Many thanks also to $\mathrm{Dr} A$ Elkind and $\operatorname{Dr} A$ Eardley for their help and advice throughout the course of the study.

1 Lancaster GA, Elkind A, Eardley A. Monitoring computermanaged cervical cytology screening in Manchester. Manchester: Department of Epidemiology and Social Oncology, Christie Hospital, 1990. (DESO 87.)

2 Eardley A, Elkind AK, Spencer B, Hobbs P, Pendleton LL, Haran D. Attendance for cervical screening-whose problem? Soc Sci Med 1985; 20: 955-62.

3 Coulter B, Baldwin A. Survey of population coverage in cervical cancer screening in the Oxford region. $\mathcal{F} R$ Coll $\mathrm{Gen}$ Pract 1987; 37: 441-3.

4 Elkind A, Eardley A, Haran D, Spencer B, Smith A. Computer-managed call and recall for cervical screening: a typology of reasons for non-attendence. Community Med typology of reason

5 Eardley A, Elkind A. A pilot study of attendance for breast cancer screening. Soc Sci Med 1990; 30: 693-9.

6 Department of Health Advisory Committee. Consolidated guidance on breast cancer screening. Oxford: NHSBSP Publications, 1990.

7 Roberts MM, Loudon NB, Huggins A. Cervical screening at a breast screening clinic. Health Bull 1988; 46: 213-6.

8 Leather DS, Roberts MM. Older women's attitudes towards breast disease, self examination, and screening facilities: implications for communication. $B M F$ 1985; 290: 668-70.

9 Holland WW, Stewart S. Screening in health care. London: The Nuffield Provincial Hospitals Trust, 1990. 